\title{
Low-Coherence Fibre-Optic Interferometric Sensors
}

\author{
M. Jedrzejewska-Szczerska, M. GnybA*, B.B. Kosmowski \\ Department of Optoelectronics and Electronics Systems, Gdańsk University of Technology \\ G. Narutowicza 11/12, 80-233 Gdańsk Poland
}

\begin{abstract}
In this paper low-coherence fibre-optic sensors based on the Fabry-Perot and Michelson interferometer, which were designed and elaborated at our laboratory, are shown. We present results of investigation of the fibre-optic sensors working in both configurations. These results allowed us to compare properties of sensors working in both configurations. The theoretical analysis and experimental results made us possible to select best construction, which was implemented in low-coherence fibre-optic sensors of refractive index.
\end{abstract}

PACS: 42.79.-e, 42.81.Pa

\section{Introduction}

A low-coherence fibre optic sensor consists of a broadband source, a sensing interferometer and an optical processor. Usually, superluminescent diodes (SLDs) or high power luminescent diodes are used as the sources. The sensing interferometer includes the measurand field or is located inside it. The sensing interferometer is designed in such a way that a defined relationship exists between optical path difference (OPD) and the measurand. The signal from the sensing interferometer is transmitted back by the fibre-optic to the optical processor. The optical processor is a device whose output signal is a function of the OPD existing in the sensing interferometer. In low-coherent fibre-optic interferometric sensors it is possible to use two kinds of optical signal processing: in a phase domain (also known as a temporal) and in a spectral domain. The optical processor can be either a second interferometer (when the phase domain processing of the measured signal is used) or a spectrometer (when the spectral domain processing of the measured signal is used) [1].

In our previous projects we implemented low-finesse Fabry-Perot interferometer as a sensing interferometer [2-5]. The main aim of research presented in this paper was to elaborate two low-coherent fiber-optic interferometric sensors of refractive index using spectral signal processing. Not only the device using the Fabry-Perot interferometer but the device with the Michelson interferometer was tested as well. This approach lets us compare and contrast metrological abilities and usefulness of sensors with spectral signal processing, working in both interferometer configurations.

\section{Spectral signal processing}

In case of low-coherent fibre-optic interferometric sensors using phase signal processing, the most crucial challenge is correct determination of a position of the central

\footnotetext{
* corresponding author; e-mail: marcin.gnyba@gmail.com
}

fringe in the interference fringe pattern. It is so important because this position refers to the zero value of the OPD and therefore gives information about the measurand. The intensity difference between the central fringe and the first side fringe can be so small that the signal-to-noise ratio required by the system for direct identification of the central fringe should not be much worse than $50 \mathrm{~dB}$, which is difficult to obtain in real optoelectronics systems [1].

Optical intensity at the output of such an interferometer can be expressed as [6]:

$$
I_{\mathrm{out}}=\left\langle E E^{*}\right\rangle \text {, }
$$

where $E=E_{1}+E_{2}, E 1$ and $E 2$ - amplitudes of the electric vector of the light wave reflected from the first and the second reflective surfaces inside the sensing interferometer respectively, brackets \langle\rangle denote time averages; asterisk $*$ denotes the complex conjugation.

When the spectral signal processing is used, the recorded signal can be expressed as [7]:

$$
I_{\text {out }}(\nu)=S(\nu)\left[1+V_{0} \cos (\Delta \phi(\nu))\right],
$$

where $S(\nu)$ - spectral distribution of the light source; $V_{0}$ — visibility of the measured signal, $\Delta \phi(\nu)$ - the phase difference between interfering beams [1].

The phase difference between interfering beams can be calculated from a following equation:

$$
\phi(\nu)=\frac{2 \pi \nu \mathrm{OPD}}{c},
$$

where OPD - the optical path difference, $c$ - light velocity in the vacuum.

If a broadband source such as SLD or laser diode (LD) working below lasing threshold is used in the sensor, complex degree of temporal coherence (or normalized degree of autocorrelation) usually has a Gaussian profile determined by the form of spontaneous emission. In this case the normalized spectra pattern is predicted to be a cosine function modified by the Gaussian visibility profile, as shown in Fig. 1.

In the spectral domain signal processing, the modulation frequency of the measurement signal gives informa- 


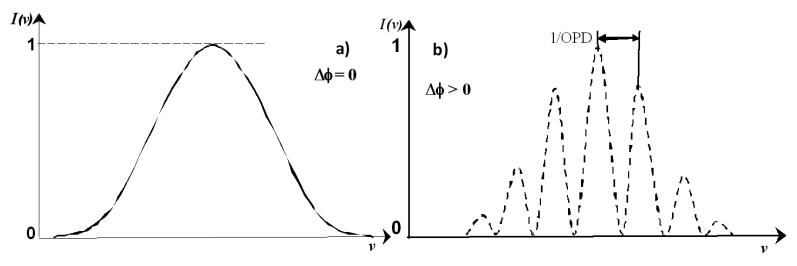

Fig. 1. The signal of low-coherence system with spectral signal processing: (a) when $\Delta \phi=0$, (b) when $\Delta \phi>0 ; \mathrm{OPD}$ - the optical path difference.

tion about the measurand as it is shown in Fig. 1. It can be noted that if $\Delta \phi=0$, then there is no spectral modulation (Fig. 1a). If the phase difference between the interfering beams varies from zero, then the function takes the form of the cosine curve (Fig. 1b). The spacing of adjacent transmission peak is proportional to the inverse of the optical path difference (1/OPD) [8]. In this type of the signal processing it is necessary to use special measurement equipment and mathematical processing of the measurement signal, which makes this method relatively expensive, complex and time-consuming. Moreover, the measurement system with the phase domain processing of the measured signal has much higher measurement sensitivity and resolution than in case of the system with spectral processing. However, the system with the spectral processing of the measured signal has two important advantages. It does not need movable mechanical components which adjust precisely displacements. Moreover, it is not sensitive for any change of a transmission of the optical system. This is possible because in the system information about the measurand is encoded in the spectra of the measured signal. Therefore the signal processing in spectral domain can be successfully used in low-coherence sensors [9].

\section{Experimental setup}

The scheme of the developed low-coherence fiber-optic interferometric sensors is shown in Fig. 2. These sensors were elaborated with the use of the Fabry-Perot interferometer (Fig. 2a) and the Michelson interferometer (Fig. 2b). The sensor head (sensing interferometer) generates interference signal which is transmitted back to optical spectrum analyzer (Ando AQ6319) through a fiber coupler. The superluminescent diode with Gaussian spectral intensity distribution (Superlum Broadlighter S1550-G-I-10 with following optical parameters: $\lambda=1560 \mathrm{~nm}, \Delta \lambda=50 \mathrm{~nm})$ is used as a low-coherence source.

The Fabry-Perot interferometer was built using standard single mode telecommunication optical fibres, a fibre coupler, the measurand field and a mirror [9]. When the measurand field is filled by the investigated liquid, the reflective surfaces of the Fabry-Perot are made by two boundaries: fibre/liquid and liquid/mirror, respectively. In such a setup each change of refractive index of the

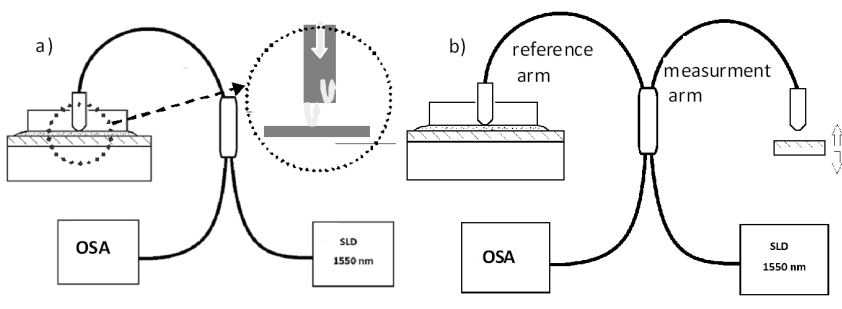

Fig. 2. The experimental setups with interferometers in (a) the Fabry-Perot configuration and (b) the Michelson configuration.

investigated liquid results in change of the OPD of interfering beams and the phase difference of those beams due to Eqs. (2) and (3).

The Michelson interferometer was elaborated with the use of standard single mode telecommunication optical fibres and fibre-optic coupler. Investigated samples were introduced on silver mirror. The position of the other silver mirror, placed in the reference arm was adjustable due to application of precise micromechanical components.

\section{Measurements of refractive index}

With the use of elaborated sensors with the FabryPerot and Michelson interferometer, the refractive index of numerous liquids was measured, for example: acetone with refractive index $n=1.358$, butyl acetate $n=1.394$ and benzyl alcohol $n=1.546$. Experimental investigation gave a series of recorded spectra. In Fig. 3 measured reference signal from sensor without any liquid is presented. In Fig. 3a signal from the sensor with the Fabry-Perot interferometer is shown, while in Fig. $3 \mathrm{~b}-$ from the sensor with the Michelson interferometer, respectively. Furthermore, in Fig. 4 and Fig. 5 the measured signal is presented for butyle acetate and benzyl alcohol, respectively.

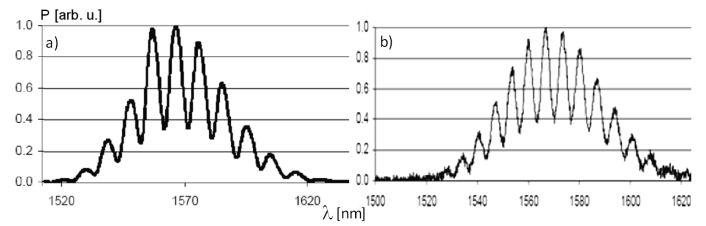

Fig. 3. Measured signal of air $(n=1)$ from the sensor with interferometer: (a) in the Fabry-Perot configuration, (b) the Michelson configuration.

Measured signals were analysed and by the use of mathematical treatment it was able to obtain information about measurand from the spectrum of signal.

The result of experimental works show that can be seen in Fig. 6 that sensors with both interferometer configurations have proper ability to measure the refractive 


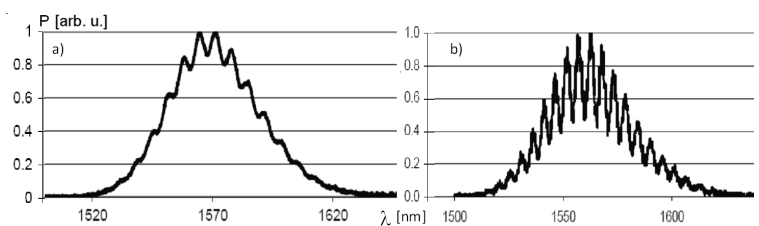

Fig. 4. Measured signal of the butyle acetate $(n=$ 1.394) from the sensor with interferometer in: (a) the Fabry-Perot configuration, (b) the Michelson configuration.

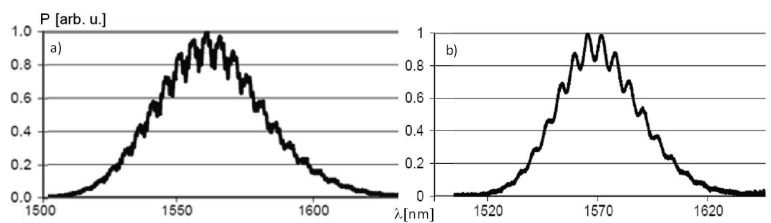

Fig. 5. Measured signal of the benzyl alcohol $(n=$ 1.546) from the sensor with interferometer in: (a) the Fabry-Perot configuration, (b) the Michelson configuration.

index. The experimental investigation made able to compare and contrast metrological parameters of both sensors, which has been summarized in Table.

Taking into consideration the value of metrological parameters of both sensor configuration, it can be noted that the exploitation of the Michelson interferometer configuration lead to meaningful increase in the value of contrast (visibility) of the measured signal (usually 0.6), which always leads to decrease of the value of signal-to-noise ratio of the measured signal. Moreover, the use of such a configuration of sensor results in good sensitivity (3.7 nm/a.u.), low measurement uncertainty $( \pm 1.66 \%)$ and very high determination coefficient of measured value (0.99). However, its calibration is complicated and time-

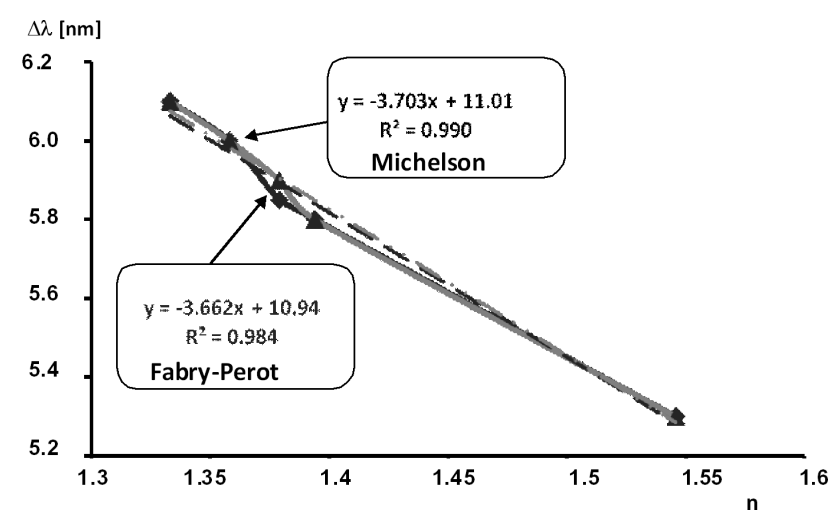

Fig. 6. The change of difference between positions of neighbouring maxima $\Delta \lambda$ in the spectra vs. refractive index $n$ : solid line - measured value, dash line - red line, black lines - the sensor with the Fabry-Perot interferometer, grey - the sensor with the Michelson interferometer. -consuming as well as degree of set-up complication is high.

Metrological parameters of the sensors.

TABLE

\begin{tabular}{l|c|c}
\hline \hline \multicolumn{1}{c|}{ Sensitivity [nm/a.u.] } & Michelson & Fabry-Perot \\
\hline determination coefficient & 3.70 & 3.67 \\
determination coefficient & 0.99 & 0.98 \\
mesurement uncertainty [\%] & \pm 1.66 & \pm 1.81 \\
contrast of measured signals & 0.6 (usually) & 0.2 (usually) \\
degree of the complexity of the setup & high & low \\
calibration & difficult & simple
\end{tabular}

Sensor with the Fabry-Perot interferometer configuration has almost similar value of determination coefficient of measured value (0.98), but lower value of contrast (visibility) of the measured signal (usually 0.2), smaller sensitivity $(3.67 \mathrm{~nm} /$ a.u. $)$ and higher measurement uncertainty $( \pm 1.81 \%)$. However, its calibration is easy and degree of setup complication is low.

\section{Conclusions}

In this paper the fiber-optic refractive index sensors using low-coherence interferometer in the Fabry-Perot and Michelson configurations have been presented. Elaborated sensors have numerous advantages: relatively simple configuration, potentially low cost, high resolution and low thermal inertia, small size of optical sensor heads, dielectric construction. Furthermore, by utilizing spectral signal processing those sensors exhibit immunity of any changes of optical transmission in measurement system.

The results of experimental works showed that the Michelson interferometer provided better quality of the measured optical signals by offering greater value of visibility of the measured signal. However the Fabry-Perot interferometer had simpler configuration and exhibits immunity for changes of the optical signal polarization.

The investigation of these sensors have shown its ability for the refractive index control with appropriate measurement parameters. Presented preliminary results can be the base for building refractive index sensor ready for practical applications.

\section{Acknowledgments}

This study was partially supported by the Polish Ministry of Science and Higher Education under the grant No. N N515 335636 and by the European Regional Development Fund in frame of the project: UDA-POIG.01.03.01-22-139/09-02 "Home assistance for elders and disabled - DOMESTIC", Innovative Economy 2007-2013, National Cohesion Strategy as well as by DS fundings of the Faculty of Electronics, Telecommunications and Informatics of the Gdańsk University of Technology. 


\section{References}

[1] K.T.V. Grattan, B.T. Meggit, Optical Fiber Sensor Technology, Kluwer Academic Publisher, Boston 2000.

[2] M. Jędrzejewska-Szczerska, R. Hypszer, Proc. SPIE 5505, 72 (2004).

[3] M. Jędrzejewska-Szczerska, R. Hypszer, in: Book of Abstracts, 16th IMEKO TC-4 Symp., Florence (Italy), Vol. 20, 2008, p. 73.

[4] E. Maciak, Z. Opilski, T. Pustelny, M. Bednorz, J. Phys. IV (France) 129, 131 (2005).
[5] Fiber Optic Sensors, Ed. F. Yu, Marcel Dekker, New York 2002

[6] S. Egerov, A. Mamaev, I. Likhachiev, Proc. SPIE 2594, 193 (1996).

[7] P. Hlubina, P. Stejskal, Proc. SPIE 4517, 267 (2001).

[8] M. Jędrzejewska-Szczerska, R. Bogdanowicz, M. Gnyba, R. Hypszer, B.B. Kosmowski, Eur. Phys. J. Spec. Top. 107, 154 (2008).

[9] B. Yu, D.W. Kim, J. Deng, H. Xiao, A. Wang, Appl. Opt. 42, 3241 (2003). 